\title{
RRM2 elicits the metastatic potential of breast cancer cells by regulating cell invasion, migration and VEGF expression via the PI3K/AKT signaling
}

\author{
SUJING ZHUANG ${ }^{1}$, LI LI ${ }^{1}$, YUWEI ZANG ${ }^{2},{\text { GUANGFENG } \mathrm{LI}^{1 *} \text { and FENG WANG }}^{3 *}$ \\ ${ }^{1}$ Department of Neurology, Linyi Central Hospital; ${ }^{2}$ Department of Radiology, Yishui People's Hospital, \\ Linyi, Shandong 276499; ${ }^{3}$ Department of Breast Disease, Beijing Tiantan Hospital, Capital Medical University, \\ Beijing 100050, P.R. China
}

Received March 29, 2019; Accepted August 30, 2019

DOI: $10.3892 / \mathrm{ol} .2020 .11428$

\begin{abstract}
Breast cancer is the second leading primary cause for cancer-related mortality among women and metastasis to the brain is a disastrous event for patients with increasing incidence. A previous study confirmed the critical function of RRM2 in breast cancer cell growth. Unfortunately, the role and fundamental molecular mechanism of RRM2 in breast cancer metastasis remains elusive. In the current study, higher RRM2 expression was validated in breast cancer tissues, especially in the brain metastasis group. Simultaneously, the expression of RRM2 was increased in breast cancer cells relative to the normal breast epithelial cell line MCF-10A, concomitant with higher levels of RRM2 in the highly metastatic MDA-MB-231 cell line relative to the weakly metastatic MCF-7 cell line. Knockdown of RRM 2 by small interfering-RRM 2 transfection notably suppressed the malignant metastatic behavior of breast cancer cells, including invasion and migration. Simultaneously, RRM2 downregulation also restrained the transcription and release of vascular endothelial growth factor (VEGF) in breast cancer cells. Moreover, inhibition of RRM2 dampened the activation of phosphatidylinositol 3 kinase (PI3K)/protein kinase B (AKT) signaling by decreasing phosphorylated-AKT and downstream matrix metalloproteinases-2 expression.
\end{abstract}

Correspondence to: Professor Feng Wang, Department of Breast Disease, Beijing Tiantan Hospital, Capital Medical University, 6 Tiantanxili Road, Beijing 100050, P.R. China

E-mail: wangfengedu1234@163.com

Dr Guangfeng Li, Department of Neurology, Linyi Central Hospital, 17 Jiankang Road, Linyi, Shandong 276499, P.R. China

E-mail: liguangfengedu1@outlook.com

*Contributed equally

Key words: breast cancer, brain metastasis, RRM2, cell invasion and migration, vascular endothelial growth factor, phosphatidyl inositol 3 kinase/protein kinase B
Intriguingly, reactivation of the PI3K/AKT pathway with its agonist insulin-like growth factor-1 reversed the adverse effects of RRM2 suppression on cancer cell invasion, migration and VEGF expression. Together, these findings suggest that RRM2 may act as a pro-metastatic factor to facilitate breast cancer metastasis by evoking cell invasion, migration and VEGF expression through the PI3K/AKT signaling pathway. This study may provide an attractive target for metastatic intervention in breast cancer.

\section{Introduction}

Breast cancer represents the second leading cause of cancer-associated mortality among women and accounts for $25.1 \%$ of all carcinomas around the world (1). Breast cancer affects $\sim 1.2$ million women worldwide each year and exhibits higher incidence in developed countries, including the USA (2). Breast cancer constitutes a global threat to public health due to its rapidly increasing prevalence and mortality. Decades of research have successfully broadened multidisciplinary therapeutic strategies for those diagnosed with breast cancer, including microsurgical removal and radiochemotherapy. Unfortunately, the survival of patients who underwent surgery for breast cancer is still limited to several months due to the high incidence of tumor cell metastasis to distant organs $(3,4)$. A total of $\sim 15-25 \%$ of breast cancer patients with brain metastasis will develop into central nervous system metastases, leading to a poor 1-year survival rate of $20 \%$ (3). Therefore, better understanding of the mechanism responsible for the progression of breast cancer metastasis is urgently needed to develop a more effective therapeutic strategy.

Ribonucleotide reductase (RR) is a rate-limiting enzyme used to induce 2'-deoxyribonucleoside 5'-diphosphates that is essential for DNA replication and repair. RRM2 is a critical RR subunit and has received significant attention in carcinoma research because its expression is dysregulated in multiple cancer types, including breast cancer $(5,6)$. Patients with high RRM2 often suffer from poor prognoses and tumor recurrence in several cancers, such as non-small cell lung cancer, colorectal cancer, adrenocortical cancer and breast cancer (5-8). RRM2 can act as a proliferation-related oncogene 
and its overexpression is positively correlated with higher grade in cancers $(5,9)$. A recent study has focused on the function of RRM2 in breast cancer growth. For instance, upregulation of RRM 2 by DSCAM-AS1 enhances breast cancer cell proliferation and inhibits apoptosis (10). Additionally, knockdown of RRM2 overturns protein kinase B (AKT)-induced tamoxifen resistance in breast cancer cells by regulating cell growth and DNA damage (11). Until now, information concerning the roles of RRM2 in breast cancer metastasis has been unavailable.

Given that the previous evidence corroborates the fact that RRM2 elevation is positively correlated with invasion depth, distant metastasis and tumor node metastasis stage in patients with colorectal cancer, indicating a pro-metastatic potential of RRM2 in colorectal cancer $(8,12)$. Therefore, in the current study the expression of RRM2 in breast cancer tissues with metastasis and cells with various metastatic ability was detected. Furthermore, the effects of RRM2 knockdown on cell invasion, migration and VEGF expression were also investigated.

\section{Materials and methods}

Clinical specimens and ethics statement. Breast cancer tissues and matched adjacent normal specimens were enrolled and collected from 25 female patients with breast cancer (age range, 28-67 years; median age, 52 years), including 10 cases with brain metastasis. Samples were surgically resected from patients who were diagnosed with breast cancer in the Department of Breast Disease of Beijing Tiantan Hospital, Capital Medical University between January 2015 and December 2017. None of patients underwent systemic anti-carcinoma therapy before surgical treatment. All tissue acquisitions were approved by the Research Ethics Committee of the Beijing Tiantan Hospital, Capital Medical University and conducted according to the Declaration of Helsinki. Written informed consent was collected from all participants. The obtained samples were preserved in liquid nitrogen and stored at $-80^{\circ} \mathrm{C}$ until processing.

Antibodies. Mouse monoclonal antibodies to human RRM2 (cat. no. ab57653) and rabbit monoclonal antibodies to human MMP-2 antibodies (cat. no. ab92536) were purchased from Abcam. Antibodies against human phosphorylated-AKT (p-AKT; cat. no. 4060) and AKT (cat. no. 9272) were obtained from acquired from Cell Signaling Technology, Inc.

Cell culture. Human breast cancer cell lines (MCF-7 and MDA-MB-231) and the normal breast epithelial cell line MCF-10A were purchased from the American Type Culture Collection. For culture, all cells were incubated in Dulbecco's modified Eagle medium (Gibco; Thermo Fisher Scientific, Inc.) supplemented with $10 \%(\mathrm{v} / \mathrm{v})$ fetal bovine serum (HyClone; GE Healthcare Life Sciences) and 1X penicillin/streptomycin (Gibco; Thermo Fisher Scientific, Inc.). Cells were maintained in a humidified incubator with $95 \%$ air and $5 \% \mathrm{CO}_{2}$ at $37^{\circ} \mathrm{C}$.

Knockdown of RRM2 by small interfering RNA interference (siRNA). To silence the expression of RRM2, oligonucleotide sequences (Gene ID 6241) that target RRM2 (si-RRM2; cat. no. sc-36338) and scrambled siRNA (cat. no. sc-37007; used as a negative control; si-NC) were applied and purchased from Santa Cruz Biotechnology, Inc. For siRNA transfec-tion experiments, MDA-MB-231 cells $\left(5 \times 10^{5}\right.$ cells per well) were seeded into 6 -well plates. Then, cells were transfected with $100 \mathrm{nM}$ siRNA using Lipofectamine RNAiMAX reagent (Invitrogen; Thermo Fisher Scientific, Inc.). Approximately forty-eight hours later, the expression of RRM2 was evaluated by reverse transcription quantitative (RT-q)PCR and western blotting analysis.

RNA extraction and RT-qPCR. Total RNA from breast cancer tissue specimens and cells was extracted using the TRIzol reagents (Thermo Fisher Scientific, Inc.). Subsequently, the prepared RNA was used to synthesize the first strand cDNA in accordance with the protocol of RevertAid First Strand cDNA Synthesis kit (Thermo Fisher Scientific, Inc.). All reactions to prepare cDNA were performed at $42^{\circ} \mathrm{C}$ for $1.5 \mathrm{~h}$. Then, $\mathrm{qPCR}$ was performed in triplicate using a SYBR Premix Ex Taq $^{\mathrm{TM}}$ II kit (Takara Bio, Inc.) according to the manufacturers' protocol. The PCR protocol was conducted using the following conditions: $95^{\circ} \mathrm{C}$ for $30 \mathrm{sec}$, followed by 40 cycles of amplification $\left(95^{\circ} \mathrm{C}\right.$ for $5 \mathrm{sec}$ and $60^{\circ} \mathrm{C}$ for $\left.30 \mathrm{sec}\right)$. All reactions were performed with an ABI PRISM 7000 sequence detection system (Applied Biosystems). The specific primers for RRM2 amplification were used as follows: Sense, 5'-ACCTATGGT GAACGTGTTGTAG-3' and antisense, 5'-GGCATCAGT CCTCGTTTCTT-3'. $\beta$-actin was used as a reference control to normalize the transcriptional levels of target gene and data was calculated using the $2^{-\Delta \Delta \mathrm{Cq}}$ method (13).

Western blotting assay. Cells were treated with si-RRM2 or PI3K/AKT signaling activator insulin-like growth factor-1 (IGF-1) (10 nM), then were collected and rinsed with cold PBS. Subsequently, radio-immunoprecipitation assay lysis buffer (Sigma-Aldrich, Merck KGaA) was added to lyse cells for $10 \mathrm{~min}$ and the extracted protein concentration was assessed using a bicinchoninic acid protein assay kit (Beyotime Institute of Biotechnology). To separate target protein, a total of $30 \mu \mathrm{g}$ protein was loaded into each lane and subjected to $10 \%$ SDS-PAGE, followed by the transfer to PVDF membranes (EMD Millipore). Then, membranes were incubated with $5 \%$ non-fat milk for $1 \mathrm{~h}$ at room temperature to interrupt the non-specific signal. The primary antibodies against human RRM2 (1:1,500), p-AKT (1:2,000), AKT (1:1,000), MMP-2 $(1: 1,000)$ and $\beta$-actin (cat. no. ab8227; 1:5,000; Abcam) were supplemented into membranes that were further incubated overnight at $4^{\circ} \mathrm{C}$. After culture with horseradish peroxidase (HRP)-conjugated secondary antibodies (cat. no. ab6721; 1:10,000 dilution; Abcam) for $1 \mathrm{~h}$ at room temperature, the immunostained proteins were visualized with Western Blotting Luminol Reagent (Santa Cruz Biotechnology, Inc.). Densitometric analysis of binding bands was conducted using NIH IMAGE (ImageJ 2X software; National Institute of Health). To quantify protein expression, $\beta$-actin was enrolled as a loading control.

Cell invasion and migration measurement in vitro. Invasion and migration potential of breast cancer cells were assessed by Transwell system (Corning, Inc.). All protocols were 
A

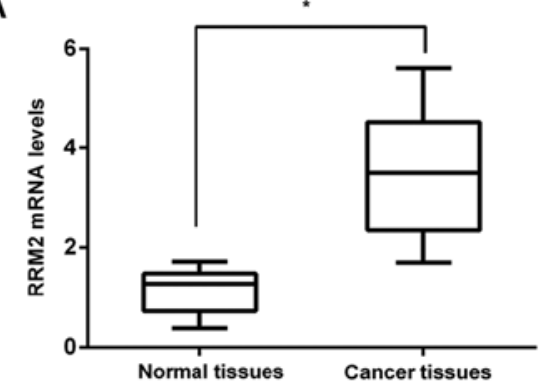

D

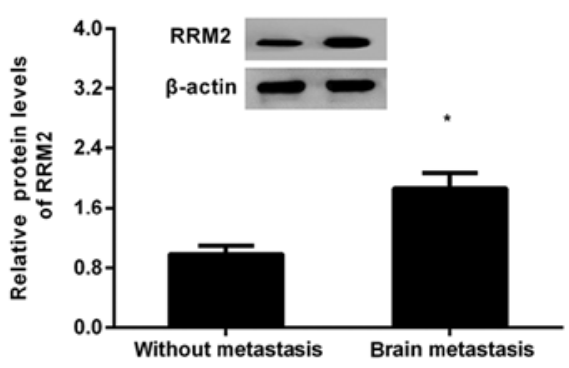

B

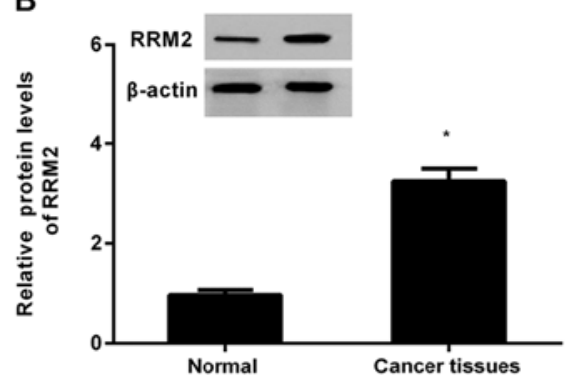

E

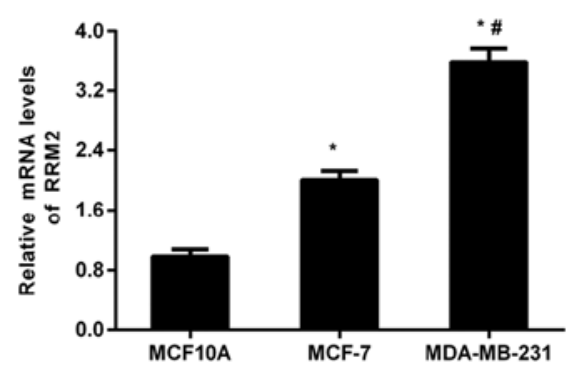

C

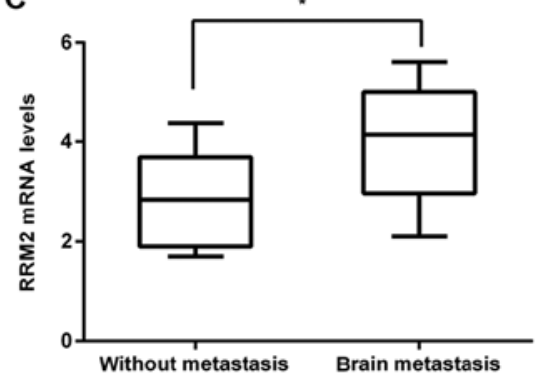

F

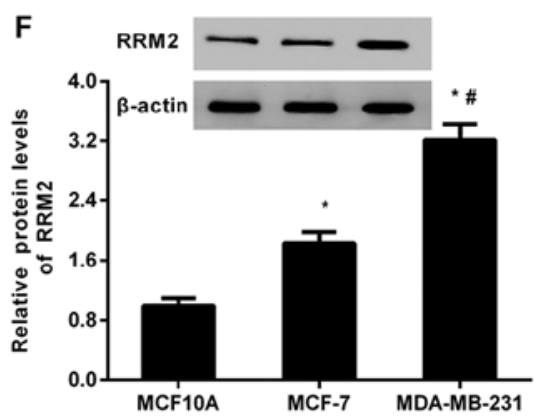

Figure 1. Expression of RRM2 is elevated in breast cancer tissues and cells. (A) The mRNA and (B) protein levels of RRM2 in normal tissues and breast cancer tissues were determined by reverse transcription-quantitative PCR and western blotting. (C) The transcript and (D) protein expression of RRM2 in cancer tissues with brain metastasis and non-metastasis groups. ${ }^{*} \mathrm{P}<0.05$. (E) mRNA and (F) protein expression of RRM2 in normal breast epithelial cell line MCF-10A, highly metastatic cell line MDA-MB-231 and weakly metastatic cell line MCF-7. ${ }^{*} \mathrm{P}<0.05$ vs. MCF-10A; ${ }^{\mathrm{P}}<0.05$ vs. MCF-7.

conducted according to the manufacturer's protocol. Briefly, for cell invasion experiments, the Transwell inserts with an $8 \mu \mathrm{m}$ pore size were pre-coated with Matrigel $(1.5 \mathrm{mg} / \mathrm{ml}$; BD Biosciences; Becton-Dickinson and Company). Then, MDA-MB-231 cells suspended in medium were seeded into the upper chamber at a density of $1 \times 10^{5}$ cells/well. Fetal bovine serum was added to lower chamber to act as a chemoattractant to induce cells through Matrigel-coated membranes. A total of $\sim 48 \mathrm{~h}$ later, non-invasive cells were removed by wiping the top of the membrane with cotton swabs. Cell migration was analyzed using the Transwell chamber without Matrigel. Then, cells that passed through the lower surface were fixed with $4 \%$ paraformaldehyde for $5 \mathrm{~min}$ at room temperature. After staining with hematoxylin for $15 \mathrm{~min}$ at room temperature, images were captured of the cells under a light microscope (magnification, $\mathrm{x} 200$ ) and 5 fields of each filter were counted to acquire an average.

ELISA detection for VEGF contents. Cells were transfected with si-RRM2 or IGF-1 and then the culture supernatants were collected. The concentration of VEGF in supernatants was determined using a commercial VEGF Quantikine ELISA kit (cat. no. SVE00; R\&D Systems, Inc.). All assays were conducted according to the company's protocol.

Statistical analysis. Data from at least three independent experiments were analyzed by SPSS 19.0 (IBM, Corps) and are presented as the mean \pm standard deviation. Differences were assessed for significance using Student t-test for two groups and one-way analysis of variance for three or more groups, followed by the Student-Newman-Keuls post hoc tests. $\mathrm{P}<0.05$ was considered to indicate a statistically significant difference.

\section{Results}

Expression of RRM2 is elevated in breast cancer tissues and cells. As presented in Fig. 1A, high expression of RRM2 mRNA was detected in breast cancer tissues relative to adjacent normal tissues, concomitant with elevation of RRM2 protein in cancer tissues (Fig. 1B). Intriguingly, higher mRNA (Fig. 1C) and protein (Fig. 1D) levels of RRM2 were observed in cancer tissues from patients with brain metastasis, compared with tissues from non-metastatic groups. Additionally, in contrast to normal breast epithelial cell line MCF-10A, RRM2 transcript levels were significantly elevated in weakly metastatic MCF-7 and highly metastatic MDA-MB-231 cells ( $\mathrm{P}<0.05$; Fig. 1E). Moreover, higher mRNA levels of RRM2 were verified in MDA-MB-231 cells compared with MCF-7 cells (Fig. 1E). There was an analogous trend in RRM2 protein expression in the breast cancer cell lines. These results may imply a possible function of RRM2 in breast cancer metastasis.

Knockdown of RRM2 suppresses the metastatic potential of breast cancer cells in vitro. To investigate the role of RRM 2 in breast cancer metastasis, the function of RRM 2 in breast cancer cell invasion and migration was elucidated. The expression of RRM2 mRNA was significantly inhibited in MDA-MB-231 cells when cells were transfected with si-RRM2 $(\mathrm{P}<0.05$; Fig. 2A). Furthermore, si-RRM2 transfection also significantly suppressed RRM2 protein expression ( $\mathrm{P}<0.05$; Fig. $2 \mathrm{~B}$ ). Simultaneously, the Transwell assay corroborated that the number of invaded cells declined to $46 \pm 11$ from $115 \pm 7$ after RRM2 knockdown (Fig. 2C). Additionally, compared with the control groups, RRM2 depression significantly inhibited cell migration ability; the number of migrated cells was reduced to 64 \pm 12 ( $\mathrm{P}<0.05$; Fig. 2D). 
A

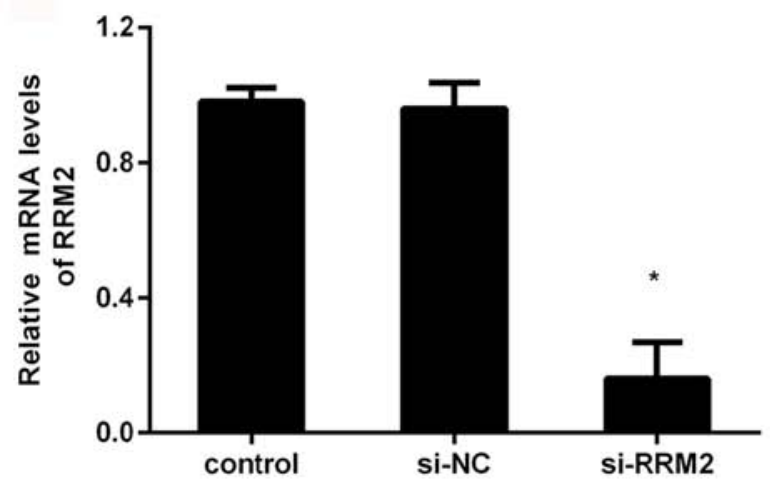

C

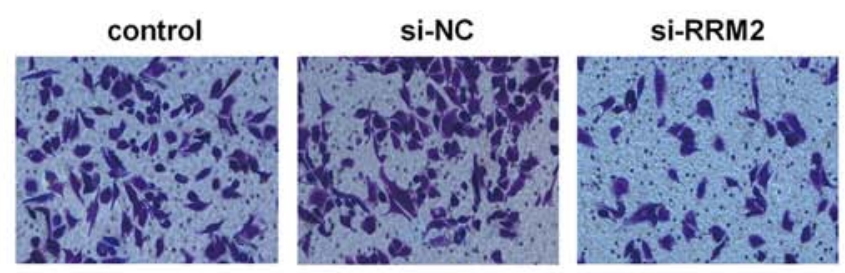

D

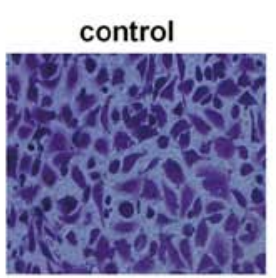

si-NC

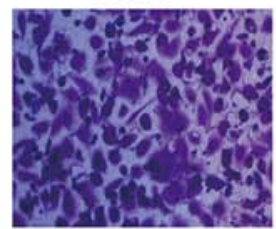

si-RRM2

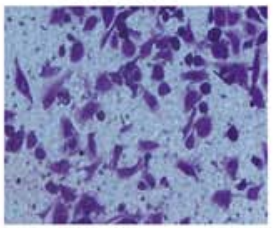

B
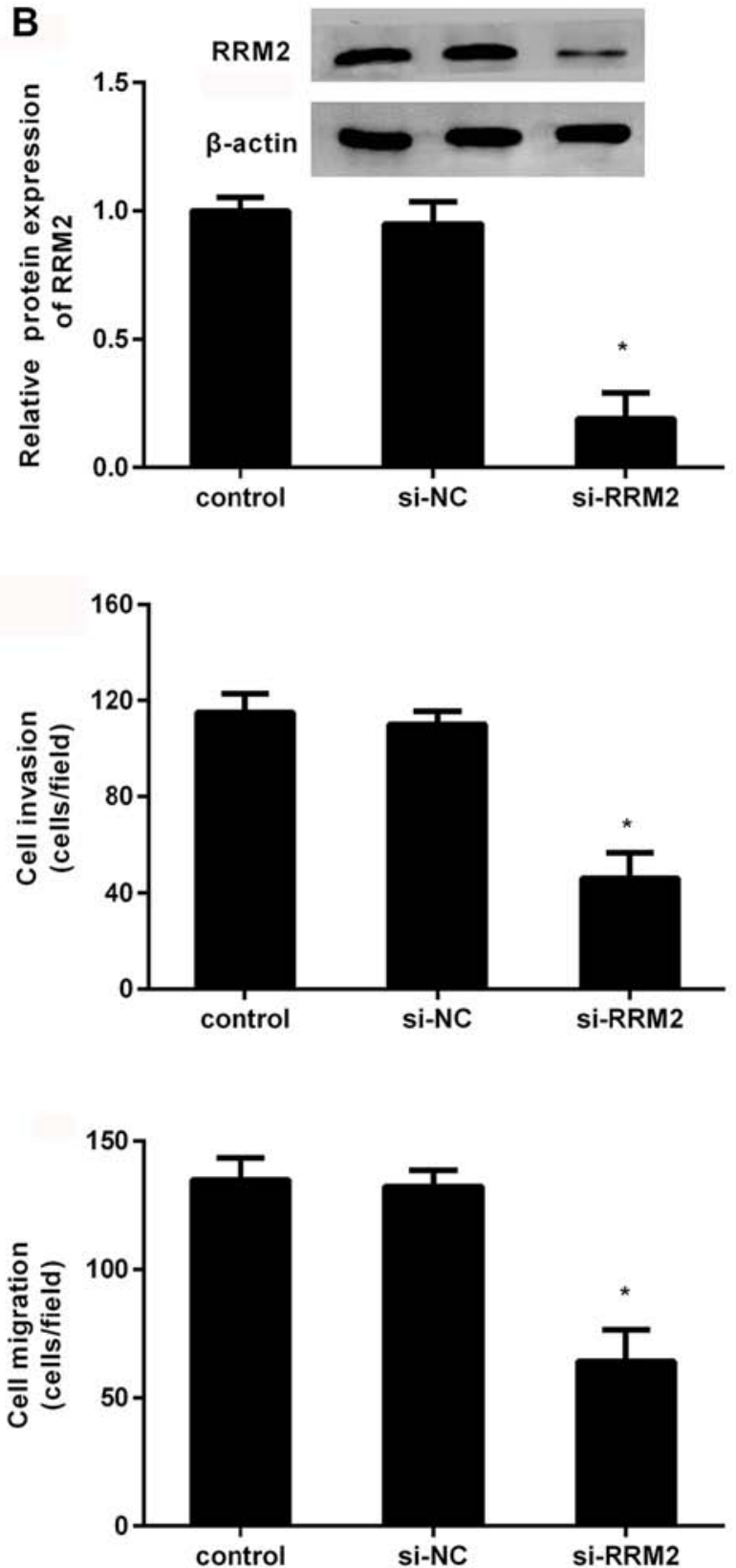

Figure 2. RRM2 knockdown restrains cell invasion and migration in MDA-MB-231 cells. (A) Breast cancer MDA-MB-231 cells were transfected with si-RRM2 or si-NC, then the mRNA of RRM2 was analyzed by reverse transcription-quantitative PCR. (B) The effects on RRM2 protein expression was detected by western blotting. After transfection with si-RRM2, (C) cell invasion and (D) migration were evaluated by Transwell assay, and images were captured under a light microscope (magnification, $\mathrm{x} 200$ ). ${ }^{*} \mathrm{P}<0.05$ vs. the control group. si, small interfering; NC, negative control.

RRM2 suppression inhibits the transcript and release of $V E G F$ in breast cancer cells. VEGF usually acts as a pivotal participator for angiogenesis that is essential for tumor growth and metastatic processes. Further evaluation confirmed that knockdown of RRM2 depressed the mRNA levels of VEGF in MDA-MB-231 cells, compared with the control and si-NC groups (Fig. 3A). Concomitantly, the contents of VEGF in the supernatants declined to $3.68 \pm 0.38 \mathrm{ng} / \mathrm{ml} / \mathrm{mg}$ when cells were transfected with si-RRM2 (Fig. 3B).

Depression of RRM2 restrains the activation of PI3K/AKT signaling. Aberrant activation of PI3K/AKT pathway has been confirmed to be implicated in cancer metastasis. To elucidate the mechanism behind the RRM2-mediated metastatic potential of breast cancer cells, the effects on PI3K/AKT signaling were investigated. As shown in Fig. 4A, depression of RRM2 inhibited the expression of p-AKT, but not the expression of AKT. Moreover, the expression of the downstream protein MMP-2 was also dampened following RRM2 knockdown. These findings suggest that RRM2 suppression abrogates the activation of the PI3K/AKT pathway in breast cancer cells.

Reactivating the PI3K/AKT pathway overturns the inhibitory effects of RRM2 deletion on metastatic potential in breast cancer cells. To further clarify the correlation between PI3K/AKT signaling and RRM2 in breast cancer cells, cells were preconditioned with the PI3K/AKT pathway 

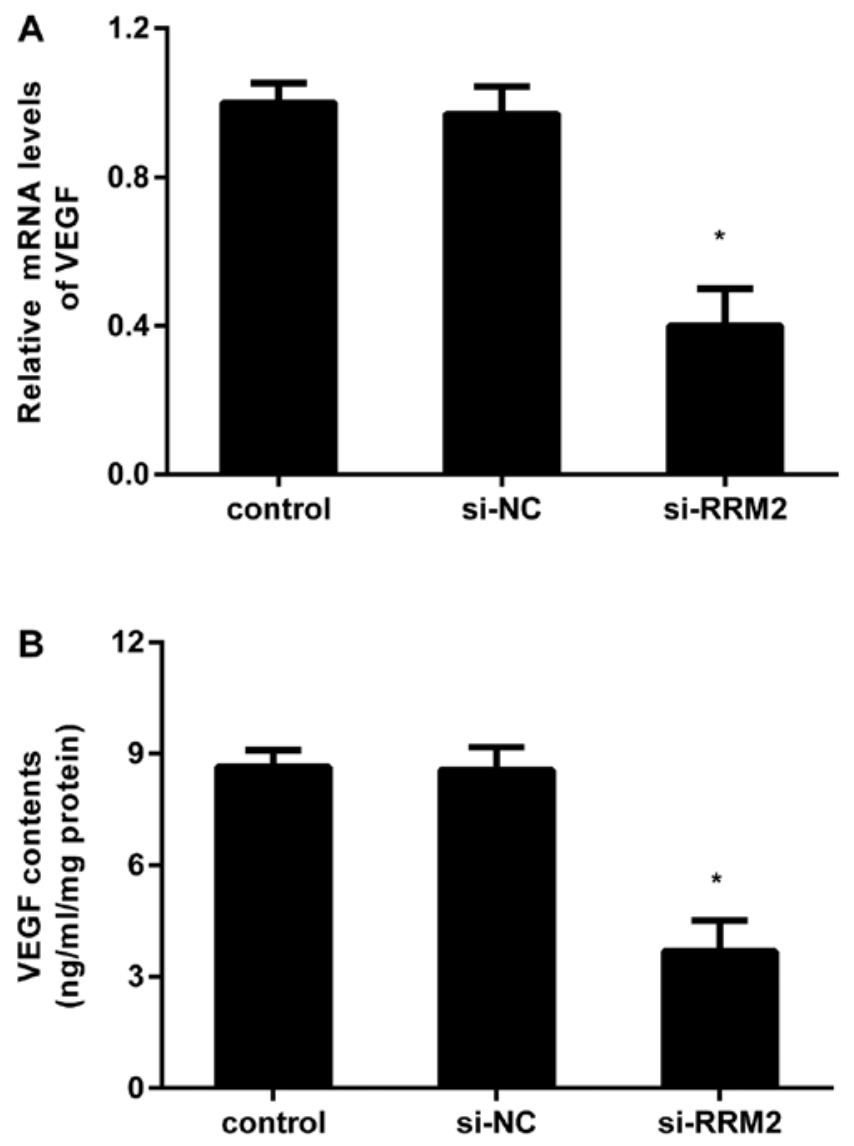

Figure 3. Depression of RRM2 suppresses the transcription and release of VEGF in breast cancer cells. (A) The si-RRM2 was transfected into MDA-MB-231 cells and the mRNA levels of VEGF was then assessed by reverse transcription-quantitative PCR. (B) The subsequent effects on VEGF contents in supernatant were measured by ELISA kits. ${ }^{*} \mathrm{P}<0.05$ vs. the contro group. si, small interfering; NC, negative control; VEGF, vascular endothelial growth factor.

agonist IGF-1. The western blotting assay confirmed that IGF-1 pretreatment restored the expression of p-AKT in RRM2-silenced breast cancer cells (Fig. 4C). Intriguingly, the suppressive function of RRM2 knockdown on breast cancer cell invasion was reversed following IGF-1 preconditioning (Fig. 4D). Simultaneously, reactivating the PI3K/AKT pathway by IGF-1 attenuated RRM2 depression-evoked inhibition in cell migration (Fig. 4E). Additionally, the concentration of VEGF in the supernatant was elevated after IGF-1 pretreatment relative to the RRM2-silenced groups (Fig. 4F).

\section{Discussion}

As a common malignancy worldwide, breast cancer usually leads to over 3.7 million deaths annually in women. RR is a rate-limiting enzyme required for DNA synthesis. Accumulating evidence validates the involvement of RR in tumor progression and resistance to various stimulus including chemotherapeutic agents $(14,15)$. RRM2, a common submit of RR, has been validated to be dysregulated in several cancers including breast cancer (5-8). However, previous studies primarily focused on its effects on breast cancer cell growth $(10,11)$. Analogous with a previous study (9), the present study also verified that there was high expression of RRM2 in breast cancer tissues. Intriguingly, higher RRM2 transcripts and protein expression were determined in cancer tissues with brain metastasis relative to non-metastatic groups. Simultaneously, in contrast to normal breast epithelial cell line MCF-10A, the expression of RRM2 was increased in breast cancer cells, concomitant with higher levels of RRM2 in highly metastatic MDA-MB-231 cells compared with the weakly metastatic MCF-7 cells. Therefore, these findings prompted the present study to discover a potential function for RRM2 in the progression of breast cancer metastasis. Intriguingly, higher expression of RRM2 has been observed in colorectal cancer patients with lymph node and distant metastases, indicating the potential value of RRM2 to predict metastases (8).

Due to the high metastasis potential of malignant cells from the primary tumor to distant organs, breast cancer has become a global health threat associated with poor survival and quality of life. Brain metastasis is a disastrous event with increasing incidence and usually occurs in $25 \%$ of patients with breast cancer (3). Metastasis is a complicated multistep process involving cell invasion, migration and angiogenesis. Based on previous results $(10,11)$, the present study elucidated further the function of RRM2 on cell metastatic potential in vitro by investigating the braintropic clone of MDA-MB-231 cells. Convincing evidence has confirmed that injection of MDA-MB-231 exhibits a stronger ability to form brain rather than bone metastases (16). Importantly, knockdown of RRM2 suppressed the invasion and migration ability of MDA-MB-231 cells, indicating that RRM2 may act as an oncogene for breast cancer cell metastasis. Analogously, RRM2 transactivation by E2F1 facilitates aggressiveness of human colorectal cancer by increasing cell invasion, migration and growth (17).

Angiogenesis is defined as the physiological process that can be formed by vascular endothelial or tumor cells. An anti-angiogenic approach has been widely accepted as a most encouraging strategy to control cancer growth and metastasis $(18,19)$. VEGF is a critical driver of sprouting angiogenesis that functions by regulating vascular formation, remodeling and permeability. Aberrant expression and activation of VEGF usually occurs in most solid tumor microenvironments, including breast cancer $(20,21)$. The present study next clarified the effects of RRM2 on VEGF levels and found that RRM2 knockdown dampened the expression and release of VEGF in breast cancer cells. Intriguingly, RRM2 overexpression increases VEGF expression to facilitate the angiogenic potential of oropharyngeal carcinoma cells, ultimately enhancing the generation of more vascularized tumor xenografts (22). Notably, elevated VEGF expression enhances the ability of breast cancer cells to form brain metastases (20). Nevertheless, discontinuation of anti-VEGF therapy aggravates cancer metastasis via the revascularization mechanism (23). Therefore, RRM2 may facilitate breast cancer metastasis by regulating VEGF-dependent angiogenesis.

The mechanism underlying RRM2-mediated breast cancer cell metastatic potential was next elucidated and it was found that suppression of RRM2 antagonized the activation of canonical PI3K/AKT signaling. Overexpression of the 
A

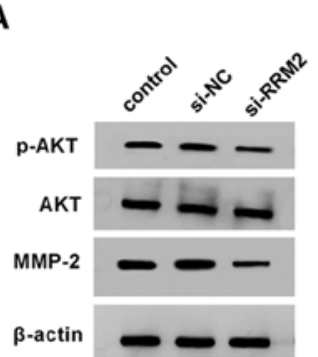
B

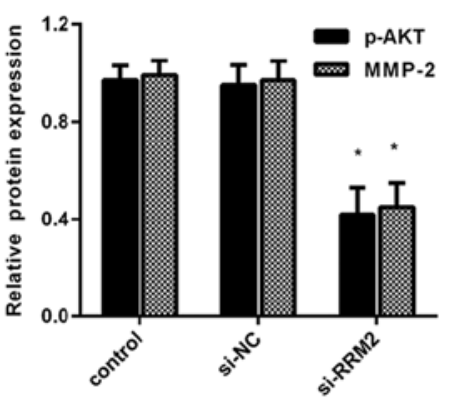

$\mathbf{F}$

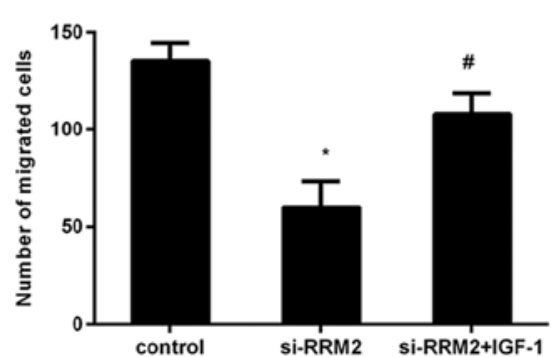

C

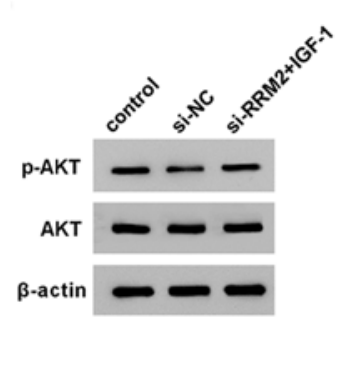

E

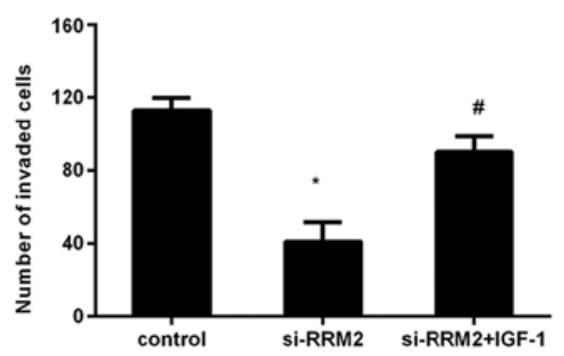

D

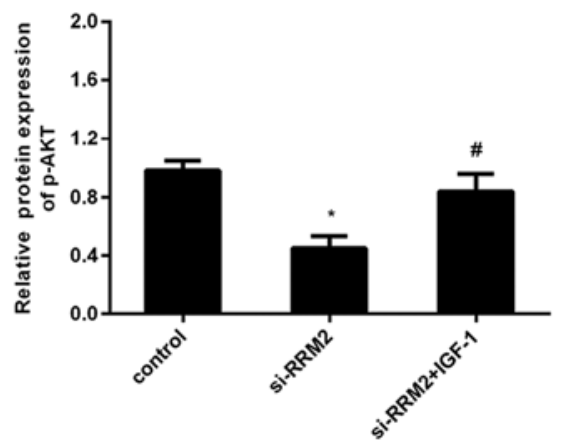

G

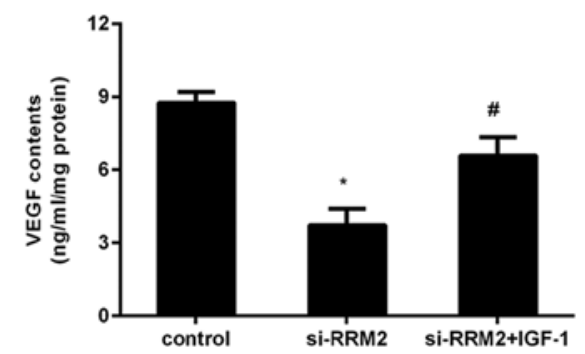

Figure 4. PI3K/AKT signaling is involved in RRM-2-mediated metastatic potential in breast cancer cells. (A) Cells were transfected with si-RRM2, protein levels of p-AKT, AKT and downstream MMP-2 were determined by western blotting. (B) The quantified analysis was performed by Image J software. (C) After preconditioning with IGF-1, cells were treated with si-RRM2. (D) Then, the expression of p-AKT was evaluated. The effects on (E) cell invasion, (F) migration and (G) VEGF production were subsequently analyzed. " $\mathrm{P}<0.05$ vs. the control group; ${ }^{\prime} \mathrm{P}<0.05$ vs. si-RRM group. si, small interfering; NC, negative control; VEGF, vascular endothelial growth factor; p-AKT, phosphorylated protein kinase B; MMP, matrix metalloproteinase; IGF-1, insulin-like growth factor-1.

PI3K/AKT axis has been substantiated in various carcinomas and possesses critical roles in carcinogenesis and drug resistance (24). Compelling research confirms that activation of $\mathrm{PI} 3 \mathrm{~K} / \mathrm{AKT}$ signaling is involved in multiple physiological processes of the carcinoma, including cancer cell proliferation, invasion, migration and apoptosis $(11,25,26)$. Inhibition of RRM2 reverses AKT-induced tamoxifen resistance by suppressing cell proliferation and motility (11). Moreover, PI3K/AKT activation enhances breast cancer invasion and metastasis (27). The involvement of PI3K/AKT and RRM2 in breast cancer metastatic potential was therefore further investigated. As expected, reactivating the PI3K/AKT pathway with its agonist IGF-1 overturned the adverse effects of RRM2 inhibition on cell invasion, migration and VEGF production in breast cancer cells. These findings suggest that PI3K/AKT activation may account for RRM2-mediated pro-metastatic function.

Collectively, the current findings corroborated the higher expression of RRM2 in breast cancer tissues with metastasis and highly metastatic cell lines. Importantly, knockdown of RRM2 restrained breast cancer cell invasion, migration and VEGF expression by regulating the PI3K/AKT signaling pathway. These data clarify a new option regarding how RRM2 facilitates breast cancer metastatic potential by enhancing cancer cell invasion, migration and angiogenesis. Therefore, RRM2 may be an attractive target for breast cancer metastatic intervention.

\section{Acknowledgements}

Not applicable.

\section{Funding}

The present study was supported by the Beijing Excellent Talents Training Assistance (Young Core Individuals; grant no. 2016000021469G211).

\section{Availability of data and materials}

All data generated or analyzed during this study are included in this published article.

\section{Authors' contributions}

FW and GL designed this manuscript. SZ and FW collected the tissues. SZ detected the expression of RRM2 in tissues and cells. LL performed western blot analysis. YZ conducted the Transwell assay. GL explored the underlying mechanism. FW wrote this manuscript. All authors read and approved the final manuscript.

\section{Ethics approval and consent to participate}

Experiments involving human participants were approved by the Research Ethics Committee of the Beijing Tiantan Hospital, Capital Medical University and conducted according to the Declaration of Helsinki. The present study followed the tenets of the Declaration of Helsinki and informed written consent was obtained from all patients.

\section{Patient consent for publication}

Not applicable. 


\section{Competing interests}

The authors declare that they have no competing interests.

\section{References}

1. Ghoncheh M, Momenimovahed $\mathrm{Z}$ and Salehiniya $\mathrm{H}$ : Epidemiology, incidence and mortality of breast cancer in Asia. Asian Pac J Cancer Prev 17: 47-52, 2016.

2. Ghoncheh M, Pournamdar Z and Salehiniya H: Incidence and mortality and epidemiology of breast cancer in the World. Asian Pac J Cancer Prev 17: 43-46, 2016.

3. Custodio-Santos T, Videira M and Brito MA: Brain metastasization of breast cancer. Biochim Biophys Acta Rev Cancer 1868 132-147, 2017.

4. Sun B, Huang Z, Wu S, Ding L, Shen G, Cha L, Wang J and Song S: Cystic brain metastasis is associated with poor prognosis in patients with advanced breast cancer. Oncotarget 7: 74006-74014, 2016.

5. Grolmusz VK, Karaszi K, Micsik T, Toth EA, Meszaros K, Karvaly G, Barna G, Szabo PM, Baghy K, Matko J, et al: Cell cycle dependent RRM2 may serve as proliferation marker and pharmaceutical target in adrenocortical cancer. Am J Cancer Res 6: 2041-2053, 2016.

6. Zhang H, Liu X, Warden CD, Huang Y, Loera S, Xue L, Zhang S, Chu P, Zheng S and Yen Y: Prognostic and therapeutic significance of ribonucleotide reductase small subunit M2 in estrogen-negative breast cancers. BMC Cancer 14: 664, 2014

7. Wang L, Meng L, Wang XW, Ma GY and Chen JH: Expression of RRM1 and RRM2 as a novel prognostic marker in advanced non-small cell lung cancer receiving chemotherapy. Tumour Biol 35: 1899-1906, 2014.

8. Chang $\mathrm{CC}$, Lin $\mathrm{CC}$, Wang $\mathrm{CH}$, Huang $\mathrm{CC}$, Ke TW, Wei PL, Yeh KT, Hsu KC, Hsu NY and Cheng YW: miR-211 regulates the expression of $R R M 2$ in tumoral metastasis and recurrence in colorectal cancer patients with a $k$-ras gene mutation. Oncol Lett 15: 8107-8117, 2018.

9. Putluri N, Maity S, Kommagani R, Creighton CJ, Putluri V, Chen F, Nanda S, Bhowmik SK, Terunuma A, Dorsey T, et al: Pathway-centric integrative analysis identifies RRM2 as a prognostic marker in breast cancer associated with poor survival and tamoxifen resistance. Neoplasia 16: 390-402, 2014.

10. Liang WH, Li N, Yuan ZQ, Qian XL and Wang ZH: DSCAM-AS1 promotes tumor growth of breast cancer by reducing miR-204-5p and up-regulating RRM2. Mol Carcinog 58: 461-473, 2019.

11. Shah KN, Mehta KR, Peterson D, Evangelista M, Livesey JC and Faridi JS: AKT-induced tamoxifen resistance is overturned by RRM2 inhibition. Mol Cancer Res 12: 394-407, 2014.

12. Lu AG, Feng H, Wang PX, Han DP, Chen XH and Zheng MH Emerging roles of the ribonucleotide reductase M2 in colorectal cancer and ultraviolet-induced DNA damage repair. World J Gastroenterol 18: 4704-4713, 2012.
13. Livak KJ and Schmittgen TD: Analysis of relative gene expression data using real-time quantitative PCR and the 2(-Delta Delta C(T)) method. Methods 25: 402-408, 2001.

14. Dai L, Lin Z, Qiao J, Chen Y, Flemington EK and Qin Z: Ribonucleotide reductase represents a novel therapeutic target in primary effusion lymphoma. Oncogene 36: 5068-5074, 2017.

15. Mannargudi MB and Deb S: Clinical pharmacology and clinical trials of ribonucleotide reductase inhibitors: Is it a viable cancer therapy? J Cancer Res Clin Oncol 143: 1499-1529, 2017.

16. El-Mabhouh AA, Nation PN, Kaddoura A and Mercer JR: Unexpected preferential brain metastases with a human breast tumor cell line MDA-MB-231 in BALB/c nude mice. Vet Pathol 45: 941-944, 2008.

17. Fang Z, Gong C, Liu H, Zhang X, Mei L, Song M, Qiu L, Luo S, Zhu Z, Zhang R, et al: E2F1 promote the aggressiveness of human colorectal cancer by activating the ribonucleotide reductase small subunit M2. Biochem Biophys Res Commun 464: 407-415, 2015.

18. Berghoff AS and Preusser M: Anti-angiogenic therapies in brain metastases. Memo 11: 14-17, 2018.

19. Viallard C and Larrivee B: Tumor angiogenesis and vascular normalization: Alternative therapeutic targets. Angiogenesis 20: 409-426, 2017.

20. Kim LS, Huang S, Lu W, Lev DC and Price JE: Vascular endothelial growth factor expression promotes the growth of breast cancer brain metastases in nude mice. Clin Exp Metastasis 21: 107-118, 2004.

21. Buijs N, Oosterink JE, Jessup M, Schierbeek H, Stolz DB, Houdijk AP, Geller DA and van Leeuwen PA: A new key player in VEGF-dependent angiogenesis in human hepatocellular carcinoma: Dimethylarginine dimethylaminohydrolase 1. Angiogenesis 20: 557-565, 2017.

22. Zhang K, Hu S, Wu J, Chen L, Lu J, Wang X, Liu X, Zhou B and Yen Y: Overexpression of RRM2 decreases thrombspondin-1 and increases VEGF production in human cancer cells in vitro and in vivo: Implication of RRM2 in angiogenesis. Mol Cancer 8: 11, 2009.

23. Yang Y, Zhang Y, Iwamoto H, Hosaka K, Seki T, Andersson P, Lim S, Fischer C, Nakamura M, Abe M, et al: Discontinuation of anti-VEGF cancer therapy promotes metastasis through a liver revascularization mechanism. Nat Commun 7: 12680, 2016

24. Guerrero-Zotano A, Mayer IA and Arteaga CL: PI3K/AKT/ mTOR: Role in breast cancer progression, drug resistance, and treatment. Cancer Metastasis Rev 35: 515-524, 2016.

25. Jin Y, Feng SJ, Qiu S, Shao N and Zheng JH: LncRNA MALAT1 promotes proliferation and metastasis in epithelial ovarian cancer via the PI3K-AKT pathway. Eur Rev Med Pharmacol Sci 21: 3176-3184, 2017.

26. Chen H, Zhou L, Wu X, Li R, Wen J, Sha J and Wen X: The $\mathrm{PI} 3 \mathrm{~K} / \mathrm{AKT}$ pathway in the pathogenesis of prostate cancer. Front Biosci (Landmark Ed) 21: 1084-1091, 2016.

27. Wu X, Sun L, Wang X, Su P, Li Z, Zhang C, Wang Y, Gao P and Ma R: Breast cancer invasion and metastasis by mPRalpha through the PI3K/Akt signaling pathway. Pathol Oncol Res 22: 471-476, 2016. 